\title{
SYMMETRIC LOCAL ALGEBRAS WITH 5-DIMENSIONAL CENTER
}

\author{
M. CHLEBOWITZ AND B. KÜLSHAMMER
}

Dedicated to Hiroyuki Tachikawa on the occasion of his sixtieth birthday

\begin{abstract}
We prove that a symmetric split local algebra whose center is 5dimensional has dimension 5 or 8 . This implies that the defect groups of a block of a finite group containing exactly five irreducible Frobenius characters and exactly one irreducible Brauer character have order 5 or are nonabelian of order 8 .
\end{abstract}

Let $F$ be a field, and let $A$ be a finite-dimensional associative unitary $F$ algebra with center $Z$ and radical $J$. Then $A$ is called split local if $\operatorname{dim} A / J=$ 1 , and $A$ is called symmetric if there is a linear map $\lambda: A \rightarrow F$ whose kernel contains all Lie commutators $[x, y]:=x y-y x \quad(x, y \in A)$ but no nonzero ideal of $A$. Suppose now that $A$ is symmetric and split local. In [6] the second author proved that $A$ is necessarily commutative if $\operatorname{dim} Z \leq 4$. This incorporated earlier results by R. Brauer and J. Brandt [1]. In this paper we are dealing with the next case.

Theorem. Let $F$ be a field, and let $A$ be a symmetric split local $F$-algebra with center $Z$. If $\operatorname{dim} Z=5$ then $\operatorname{dim} A \in\{5,8\}$.

The group algebra of a group of order 5 over a field of characteristic 5 is an example for the case $\operatorname{dim} A=5$, and the group algebra of a nonabelian group of order 8 over a field of characteristic 2 is an example for the case $\operatorname{dim} A=8$.

Corollary. Let $F$ be an algebraically closed field, let $G$ be a finite group, let $P$ be an indecomposable projective $F G$-module, and set $A:=\operatorname{End}_{F G}(P)$. If the center of $A$ has dimension 5 then $\operatorname{dim} A \in\{5,8\}$.

Proof. We choose a primitive idempotent $i$ in $F G$ such that $P$ is isomorphic to $F G i$. Then $A$ is isomorphic to $i F G i$. Since $F G$ is a symmetric $F$-algebra, so are $i F G i$ and $A$. Since $P$ is indecomposable and $F$ is algebraically closed, $A$ is split local. Hence the corollary follows from the theorem.

We have the following application to block theory.

Proposition. Let $F$ be an algebraically closed field, let $G$ be a finite group, and let $B$ be a block of $F G$ containing exactly 5 irreducible complex characters

Received by the editors September 19, 1988 and, in revised form, November 28, 1989.

1980 Mathematics Subject Classification (1985 Revision). Primary 16A36, 20C20; Secondary 16A46, 16A26, 20C05. 
and exactly one irreducible Brauer character. Then the defect groups of $B$ have order 5 or are nonabelian of order 8.

Proof. Let $P$ denote the only indecomposable projective $F G$-module in $B$, and set $A:=\operatorname{End}_{F G}(P)$. By Lemma B in [4], $B$ is isomorphic to a complete matrix algebra over $A$; in particular, $A$ and $B$ have isomorphic centers. By $(2 G)$ in [2], the dimension of the center of $B$ coincides with the number of irreducible complex characters in $B$, so the center of $A$ has dimension 5 . By the corollary, $A$ has dimension 5 or 8 . On the other hand, Lemma B in [4] shows that the dimension of $A$ coincides with the order of a defect group $D$ of $B$. Hence $D$ has order 5 or 8 . Assume now that $D$ is abelian of order 8 . Then $B$ cannot be nilpotent in the sense of [3]; for otherwise $B$ would contain 8 irreducible complex characters by the main result of [3]. Thus $D$ must be elementary abelian. But in this case we obtain a contradiction using the results in [7].

The remainder of this paper consists of a proof of the theorem. Let $A$ be a symmetric split local algebra over a field $F$ and denote by $Z$ the center and by $J$ the radical of $A$. We may and do assume that $F$ is algebraically closed. For a subset $X$ of $A$, we denote by $F X$ the linear subspace of $A$ spanned by $X$. The subspace $K:=F\{[x, y]: x, y \in A\}$ will be particularly important for us. Since $A=F 1+J$ we have $K=[J, J] \subset J^{2}$. We fix a linear map $\lambda: A \rightarrow F$ the kernel of which contains $K$ but no nonzero ideal of $A$. Then 0 is the only ideal of $A$ contained in $K$. For any linear subspace $U$ of $A, U^{\perp}:=\{a \in A: \lambda(a U)=0\}$ is a linear subspace of $A$ such that $\operatorname{dim} A=\operatorname{dim} U+\operatorname{dim} U^{\perp}$ and $\left(U^{\perp}\right)^{\perp}=U$. We have $Z^{\perp}=K$ (see [5]); in particular, $\operatorname{dim} Z=\operatorname{dim} A / K$. Moreover,

$$
I^{\perp}=\{a \in A: a I=0\}=\{a \in A: I a=0\}
$$

for any ideal $I$ of $A$; in particular, $I^{\perp}$ is an ideal of $A$. Furthermore, $\operatorname{dim} J^{\perp}=\operatorname{dim} A / J=1$. Hence, if $J^{n}=0$ for some positive integer $n$ then $J^{n-1} \subset J^{\perp}$; in particular, $\operatorname{dim} J^{n-1} \leq \operatorname{dim} J^{\perp}=1$. We will often use this fact without special reference.

\section{Preliminary Results}

From now on we suppose that $\operatorname{dim} Z=5$. We may and will assume that $\operatorname{dim} A \geq 6$; for otherwise we are done.

\section{(1.1) Lemma. We have $\operatorname{dim} A \geq 8$.}

Proof. Assume that $\operatorname{dim} A \leq 7$. Then there are elements $a, b \in A$ such that $A=Z+F a+F b$. Therefore $K=F[a, b]$; in particular, $\operatorname{dim} K \cap Z \leq \operatorname{dim} K \leq$ 1. Now Lemma $\mathrm{D}$ in [6] implies that $A$ is commutative, so $\operatorname{dim} A=\operatorname{dim} Z=$ 5 , a contradiction.

If $\operatorname{dim} A=8$, then the theorem is proved, so we may and will assume that $\operatorname{dim} A \geq 9$. We are then looking for a contradiction.

(1.2) Lemma. We have $\operatorname{dim} A / K+J^{3}=4$, and one of the following occurs:

(1.3) $\operatorname{dim} J / J^{2}=2, \operatorname{dim} J^{2} / J^{3}=2, \operatorname{dim} J^{3} / J^{4} \geq 2, \operatorname{dim} J^{4} / J^{5} \geq 1$, $K+J^{3}=K+J^{4}$ 
(1.4) $\operatorname{dim} J / J^{2}=3, \operatorname{dim} J^{2} / J^{3}=2, \operatorname{dim} J^{3} / J^{4} \geq 2, \operatorname{dim} J^{4} / J^{5} \geq 1$, $J^{2}=K+J^{3}=K+J^{4}$

(1.5) $\operatorname{dim} J / J^{2}=3, \operatorname{dim} J^{2} / J^{3}=3, \operatorname{dim} J^{3} / J^{4} \geq 2, \operatorname{dim} J^{4} / J^{5} \geq 1$, $J^{2}=K+J^{3}=K+J^{4}$.

Proof. Since $\operatorname{dim} J \geq 8$ we have $J^{2} \neq 0$. Thus Nakayama's Lemma implies that $J^{2} \neq J^{3}$. Furthermore, $J \not \subset Z$, so $\operatorname{dim} J^{2} / J^{3} \geq 2$ by Lemma $G$ in [6]; in particular, $\operatorname{dim} J / J^{2} \geq 2$ by Lemma $\mathrm{E}$ in [6], and $J^{3} \neq 0$. Hence $J^{3} \neq J^{4}$ by Nakayama's Lemma, and $J^{3} \not \subset K$. Thus

$$
\operatorname{dim} A / J^{2} \leq \operatorname{dim} A / K+J^{3}<\operatorname{dim} A / K=\operatorname{dim} Z=5 ;
$$

in particular, $\operatorname{dim} J / J^{2} \in\{2,3\}$, so $\operatorname{dim} J^{2} \geq 5$. This means that $J^{2} \not \subset Z$ which implies by Lemma $G$ in [6] that $\operatorname{dim} J^{3} / J^{4} \geq 2$. Hence $J^{4} \neq 0$, and $J^{4} \neq J^{5}$ by Nakayama's Lemma again. Moreover, $J^{4} \not \subset K$, so $\operatorname{dim} A / K+J^{3} \leq$ $\operatorname{dim} A / K+J^{4}<\operatorname{dim} A / K=5$.

Suppose first that $\operatorname{dim} J / J^{2}=2$ and write $J=F a+F b+J^{2}$ with elements $a, b \in J$. Then $A=F\{1, a, b\}+J^{2}$ and $K \subset F[a, b]+J^{3}$; in particular, $\operatorname{dim} K+J^{3} / J^{3} \leq 1$, so $\operatorname{dim} A / J^{3} \leq 5$ and $\operatorname{dim} J^{2} / J^{3}=2$. Thus $\operatorname{dim} A / J^{3}=5$ and $\operatorname{dim} A / K+J^{3}=4$. Hence also $\operatorname{dim} A / K+J^{4}=4$.

Finally, suppose that $\operatorname{dim} J / J^{2}=3$ and write $J=F\{a, b, c\}+J^{2}$ with elements $a, b, c \in J$. Then $K \subset F\{[a, b],[a, c],[b, c]\}+J^{3}$; in particular, $\operatorname{dim} K+J^{3} / J^{3} \leq 3$. Thus $\operatorname{dim} A / J^{3} \leq 7$ and $\operatorname{dim} J^{2} / J^{3} \in\{2,3\}$. Since $4=\operatorname{dim} A / J^{2} \leq \operatorname{dim} A / K+J^{3} \leq \operatorname{dim} A / K+J^{4} \leq 4$ the result follows.

We will deal with these cases in $\S \S 2,3$ and 4 , respectively. The following results will be useful later on.

(1.6) Lemma. There is an element $x \in J$ such that $x^{2} \notin J^{3}$.

Proof. By (1.2) we have $\operatorname{dim} J / J^{2} \leq 3$. We write $J=F\{a, b, c\}+J^{2}$ with elements $a, b, c \in J$. If $x^{2} \in J^{3}$ for $x \in J$ then $a b+b a=(a+b)^{2}-a^{2}-b^{2} \in$ $J^{3}$. Thus $b a \equiv-a b\left(\bmod J^{3}\right)$. Similarly, $c a \equiv-a c\left(\bmod J^{3}\right)$ and $c b \equiv-b c$ $\left(\bmod J^{3}\right)$. Therefore

$$
J^{2}=F\left\{a^{2}, a b, a c, b a, b^{2}, b c, c a, c b, c^{2}\right\}+J^{3}=F\{a b, a c, b c\}+J^{3} ;
$$

in particular, $\operatorname{dim} J^{2} / J^{3} \leq 3$. Now we apply Lemma $E$ in [6] to obtain

$$
J^{3}=F\left\{a^{2} b, a^{2} c, a b c, b a b, b a c, b^{2} c\right\}+J^{4}=F a b c+J^{4}
$$

and $J^{4}=F a^{2} b c+J^{5}=J^{5}$ contradicting (1.2).

(1.7) Lemma. There are elements $a, b \in J$ such that $a^{2}+J^{3}, a b+J^{3}$ or $a^{2}+J^{3}, b a+J^{3}$ are linearly independent in $J^{2} / J^{3}$.

Proof. By (1.6), there is an element $a \in J$ such that $a^{2} \notin J^{3}$; in particular, $a \notin J^{2}$. By (1.2) there are therefore elements $b, c \in J$ such that $J=F\{a, b, c\}+J^{2}$. We may assume that $a b, b a, a c, c a \in F a^{2}+J^{3}$; for otherwise the result is proved. Then $K+J^{3}=F\{[a, b],[a, c],[b, c]\}+J^{3} \subset$ $F\left\{a^{2},[b, c]\right\}+J^{3}$; in particular, $\operatorname{dim} K+J^{3} / J^{3} \leq 2$. Hence, by (1.2), $\operatorname{dim} J^{2} / J^{3}=2$.

Now consider the case where $b^{2} \notin F a^{2}+J^{3}$; in particular, $b^{2} \notin J^{3}$. Then we can interchange the roles of $a$ and $b$ and therefore assume that 
$a b, b a, b c, c b \in F b^{2}+J^{3}$. Since $a^{2}+J^{3}$ and $b^{2}+J^{3}$ form a basis of $J^{2} / J^{3}$ this implies that $a b, b a \in J^{3}$. Thus $(a+b)^{2}+J^{3}=a^{2}+b^{2}+J^{3}$ and $(a+b) b+J^{3}=b^{2}+J^{3}$ are linearly independent, and the result follows in this case.

Therefore we may also assume that $b^{2} \in F a^{2}+J^{3}$ and, similarly, $c^{2} \in$ $F a^{2}+J^{3}$. Then

$$
J^{2}=F\left\{a^{2}, a b, a c, b a, b^{2}, b c, c a, c b, c^{2}\right\}+J^{3}=F\left\{a^{2}, b c, c b\right\}+J^{3} .
$$

Thus $J^{2}=F\left\{a^{2}, b c\right\}+J^{3}$ or $J^{2}=F\left\{a^{2}, c b\right\}+J^{3}$; we may assume that $J^{2}=F\left\{a^{2}, b c\right\}+J^{3}$. Then Lemma $\mathrm{E}$ in [6] implies that

$$
J^{3}=F\left\{a^{3}, a b c, b a^{2}, b^{2} c\right\}+J^{4}=F\left\{a^{3}, a^{2} c\right\}+J^{4}=F a^{3}+J^{4} ;
$$

in particular, $\operatorname{dim} J^{3} / J^{4} \leq 1$ contradicting (1.2).

We now choose elements $a, b \in J$ as in (1.7). By symmetry we may assume that $a b \notin F a^{2}+J^{3}$; in particular, $a \notin J^{2}$ and $b \notin F a+J^{2}$. Thus $a+$ $J^{2}, b+J^{2}$ are linearly independent in $J / J^{2}$. By (1.2), we can find an element $c \in J$ such that $J=F\{a, b, c\}+J^{2}$.

\section{THE CASE (1.3)}

In this section we use the same hypothesis and notation as before, but we assume in addition that (1.3) holds. Then $J=F a+F b+J^{2}$ and $J^{2}=$ $F a^{2}+F a b+J^{3}$. Thus Lemma E in [6] implies that $J^{3}=F a^{3}+F a^{2} b+J^{4}$, $J^{4}=F a^{4}+F a^{3} b+J^{5}$ and $J^{5}=F a^{5}+F a^{4} b+J^{6} ;$ in particular, $\operatorname{dim} J^{3} / J^{4}=2$. Thus $a^{3}+J^{4}$ and $a^{2} b+J^{4}$ form a basis of $J^{3} / J^{4}$. Moreover, $\operatorname{dim} J^{4} \geq 2$; in particular, $J^{5} \neq 0$. Thus $J^{5} \not \subset K$ and $4=\operatorname{dim} A / K+J^{4} \leq \operatorname{dim} A / K+$ $J^{5}<\operatorname{dim} A / K=\operatorname{dim} Z=5$ by (1.2). We conclude that $\operatorname{dim} A / K+J^{5}=4$. Furthermore, $A=F\left\{1, a, b, a^{2}, a b, a^{3}, a^{2} b\right\}+J^{4}$, so

$$
K \subset F\left\{[a, b],[a, a b],\left[a, a^{2} b\right],\left[b, a^{2}\right],\right.
$$

$$
\left.[b, a b],\left[b, a^{3}\right],\left[b, a^{2} b\right],\left[a^{2}, a b\right]\right\}+J^{5} .
$$

Since $J^{2}=F\left\{a^{2}, a b, a^{3}, a^{2} b\right\}+J^{4}$ there are elements $\alpha_{i}, \beta_{i}, \gamma_{i}, \delta_{i} \in F$ $(i=1,2)$ such that

$$
\begin{aligned}
b a & \equiv \alpha_{1} a^{2}+\beta_{1} a b+\gamma_{1} a^{3}+\delta_{1} a^{2} b \quad\left(\bmod J^{4}\right), \\
b^{2} & \equiv \alpha_{2} a^{2}+\beta_{2} a b+\gamma_{2} a^{3}+\delta_{2} a^{2} b \quad\left(\bmod J^{4}\right) .
\end{aligned}
$$

We have to distinguish between two cases.

Case 1. $\beta_{1} \neq 1$. In this case we set $\xi:=\alpha_{1} /\left(1-\beta_{1}\right)$ and $b^{\prime}:=b-\xi a$. Then $J=F a+F b^{\prime}+J^{2}, J^{2}=F a^{2}+F a b^{\prime}+J^{3}$ and

$$
\begin{aligned}
b^{\prime} a & \equiv b a-\xi a^{2} \equiv\left(\alpha_{1}-\xi\right) a^{2}+\beta_{1} a b \\
& \equiv\left(\alpha_{1}-\xi+\beta_{1} \xi\right) a^{2}+\beta_{1} a b^{\prime} \equiv \beta_{1} a b^{\prime} \quad\left(\bmod J^{3}\right) .
\end{aligned}
$$

Thus we may replace $b$ by $b^{\prime}$ and therefore assume that $\alpha_{1}=0$. Then

$$
\begin{aligned}
0 & \equiv\left(b^{2}\right) a-b(b a) \equiv \alpha_{2} a^{3}+\beta_{2} a b a-\beta_{1} b a b \equiv \alpha_{2} a^{3}+\beta_{1} \beta_{2} a^{2} b-\beta_{1}^{2} a b^{2} \\
& \equiv\left(\alpha_{2}-\alpha_{2} \beta_{1}^{2}\right) a^{3}+\left(\beta_{1} \beta_{2}-\beta_{1}^{2} \beta_{2}\right) a^{2} b \quad\left(\bmod J^{4}\right)
\end{aligned}
$$


and, similarly,

$0 \equiv\left(b^{2}\right) b-b\left(b^{2}\right) \equiv\left(\alpha_{2} \beta_{2}-\alpha_{2} \beta_{1} \beta_{2}\right) a^{3}+\left(\alpha_{2}+\beta_{2}^{2}-\alpha_{2} \beta_{1}^{2}-\beta_{1} \beta_{2}^{2}\right) a^{2} b \quad\left(\bmod J^{4}\right)$.

Since $a^{3}+J^{4}$ and $a^{2} b+J^{4}$ form a basis of $J^{3} / J^{4}$ we conclude that

$$
\begin{aligned}
& 0=\alpha_{2}-\alpha_{2} \beta_{1}^{2}, \\
& 0=\alpha_{2} \beta_{2}-\alpha_{2} \beta_{1} \beta_{2},
\end{aligned}
$$

(2.2) $0=\beta_{1} \beta_{2}-\beta_{1}^{2} \beta_{2}$

(2.4) $0=\alpha_{2}+\beta_{2}^{2}-\alpha_{2} \beta_{1}^{2}-\beta_{1} \beta_{2}^{2}$.

Subtracting (2.1) from (2.4) we obtain $\beta_{2}^{2}=\beta_{1} \beta_{2}^{2}$. Since $\beta_{1} \neq 1$ this implies $\beta_{2}=0$. From (2.1) we also conclude that $\alpha_{2}=0$ or $\beta_{1}^{2}=1$. We assume first that $\alpha_{2}=0$. Then

$$
\begin{aligned}
{[a, a b] } & \equiv a^{2} b-a b a \equiv\left(1-\beta_{1}\right) a^{2} b \quad\left(\bmod J^{4}\right), \\
{\left[b, a^{2}\right] } & \equiv b a^{2}-a^{2} b \equiv \beta_{1} a b a-a^{2} b \equiv\left(\beta_{1}^{2}-1\right) a^{2} b \quad\left(\bmod J^{4}\right), \\
{[b, a b] } & \equiv b a b-a b^{2} \equiv \beta_{1} a b^{2} \equiv 0 \quad\left(\bmod J^{4}\right) .
\end{aligned}
$$

This shows that $K \subset F[a, b]+F a^{2} b+J^{4}$; in particular, $\operatorname{dim} K+J^{4} / J^{4} \leq 2$. Thus $\operatorname{dim} A / J^{4} \leq 6$ by (1.2), a contradiction.

Hence we must have $\alpha_{2} \neq 0$ and $\beta_{1}^{2}=1$. Since $\beta_{1} \neq 1$ this implies $\beta_{1}=-1$ and $\operatorname{char} F \neq 2$. It is now easy to check that

$$
\begin{array}{ll}
{\left[a, a^{2} b\right] \equiv 2 a^{3} b \quad\left(\bmod J^{5}\right),} & {\left[b, a^{2}\right] \equiv-2 \delta_{1} a^{3} b \quad\left(\bmod J^{5}\right),} \\
{\left[b, a^{3}\right] \equiv-2 a^{3} b \quad\left(\bmod J^{5}\right),} & {\left[b, a^{2} b\right] \equiv\left[a^{2}, a b\right] \equiv 0 \quad\left(\bmod J^{5}\right) .}
\end{array}
$$

Thus $K \subset F\left\{[a, b],[a, a b],[b, a b], a^{3} b\right\}+J^{5} ;$ in particular, $\operatorname{dim} K+J^{5} / J^{5}$ $\leq 4$. Hence $\operatorname{dim} A / J^{5} \leq 8$ and $\operatorname{dim} J^{4} / J^{5}=1$. By Lemma $G$ in [6], this implies that $J^{3} \subset Z$; in particular, $a^{2} b \in Z$. Thus $a^{3} b \equiv a^{2} b a \equiv-a^{3} b$ $\left(\bmod J^{5}\right)$. Since $\operatorname{char} F \neq 2$ this implies $a^{3} b \in J^{5}$. Therefore

$$
K \subset F\{[a, b],[a, a b],[b, a b]\}+J^{5} ;
$$

in particular, $\operatorname{dim} K+J^{5} / J^{5} \leq 3$. Hence $\operatorname{dim} A / J^{5} \leq 7$, a contradiction.

Case 2. $\beta_{1}=1$. Assume first that $\alpha_{1}=0$. Then $[a, b] \in J^{3}$ and $K \subset J^{3}$, so $\operatorname{dim} A / K+J^{3}=\operatorname{dim} A / J^{3}=5$ contradicting (1.2). Thus we must have $\alpha_{1} \neq 0$. Now we set $a^{\prime}:=\alpha_{1} a$. Then $J=F a^{\prime}+F b+J^{2}, J^{2}=F\left(a^{\prime}\right)^{2}+F a^{\prime} b+J^{3}$ and

$$
b a^{\prime} \equiv \alpha_{1} b a \equiv \alpha_{1}^{2} a^{2}+\alpha_{1} a b \equiv\left(a^{\prime}\right)^{2}+a^{\prime} b \quad\left(\bmod J^{3}\right) .
$$

Hence we may replace $a$ by $a^{\prime}$ and therefore assume that $\alpha_{1}=1$. As in Case 1 we compute

$$
0 \equiv\left(b^{2}\right) a-b(b a) \equiv\left(\beta_{2}-2\right) a^{3}-2 a^{2} b \quad\left(\bmod J^{4}\right) .
$$

Since $a^{3}+J^{4}$ and $a^{2} b+J^{4}$ form a basis of $J^{3} / J^{4}$ this implies that char $F=2$ and $\beta_{2}=0$. Hence

$$
\begin{gathered}
{\left[a, a^{2} b\right] \equiv a^{4} \quad\left(\bmod J^{5}\right), \quad\left[b, a^{2}\right] \equiv \delta_{1} a^{4} \quad\left(\bmod J^{5}\right)} \\
{\left[b, a^{3}\right] \equiv a^{4} \quad\left(\bmod J^{5}\right), \quad\left[b, a^{2} b\right] \equiv\left[a^{2}, a b\right] \equiv 0 \quad\left(\bmod J^{5}\right) .}
\end{gathered}
$$

Therefore $K \subset F\left\{[a, b],[a, a b],[b, a b], a^{4}\right\}+J^{5}$; in particular, $\operatorname{dim} K+$ $J^{5} / J^{5} \leq 4$. Hence $\operatorname{dim} A / J^{5} \leq 8$ and $\operatorname{dim} J^{4} / J^{5}=1$. By Lemma $G$ in [6], this implies that $J^{3} \subset Z$; in particular, $a^{2} b \in Z$. Thus $a^{3} b \equiv a^{2} b a \equiv a^{4}+a^{3} b$ 
$\left(\bmod J^{5}\right)$. Therefore $a^{4} \in J^{5}$ and $J^{5}=F a^{5}+F a^{4} b+J^{6}=J^{6}$. Hence $J^{5}=0$ by Nakayama's Lemma, a contradiction.

\section{THE CASE (1.4)}

In this section we assume hypothesis and notation from $\S 1$. In addition, we assume that (1.4) holds. Then $J^{2}=F a^{2}+F a b+J^{3}$ and $J^{3}=F a^{3}+F a^{2} b+J^{4}$ by Lemma $\mathrm{E}$ in [6]; in particular, $\operatorname{dim} J^{3} / J^{4}=2$. Hence $a^{3}+J^{4}, a^{2} b+J^{4}$ form a basis of $J^{3} / J^{4}$. There are elements $\alpha, \beta \in F$ such that $a c \equiv \alpha a^{2}+\beta a b$ $\left(\bmod J^{3}\right)$. Setting $c^{\prime}:=c-\alpha a-\beta b$ we then have $J=F\left\{a, b, c^{\prime}\right\}+J^{2}$ and $a c^{\prime} \equiv a c-\alpha a^{2}-\beta a b \equiv 0\left(\bmod J^{3}\right)$. Hence we may replace $c$ by $c^{\prime}$ and therefore assume that $a c \in J^{3}$. We choose elements $\alpha_{i}, \beta_{i} \in F \quad(i=$ $1,2,3,4)$ such that

$$
\begin{array}{llll}
b c \equiv \alpha_{1} a^{2}+\beta_{1} a b & \left(\bmod J^{3}\right), & c a \equiv \alpha_{2} a^{2}+\beta_{2} a b & \left(\bmod J^{3}\right), \\
c b \equiv \alpha_{3} a^{2}+\beta_{3} a b & \left(\bmod J^{3}\right), & c^{2} \equiv \alpha_{4} a^{2}+\beta_{4} a b & \left(\bmod J^{3}\right) .
\end{array}
$$

Then

$$
\begin{aligned}
& 0 \equiv(a c) a \equiv a(c a) \equiv \alpha_{2} a^{3}+\beta_{2} a^{2} b \quad\left(\bmod J^{4}\right), \\
& 0 \equiv(a c) b \equiv a(c b) \equiv \alpha_{3} a^{3}+\beta_{3} a^{2} b \quad\left(\bmod J^{4}\right), \\
& 0 \equiv(a c) c \equiv a\left(c^{2}\right) \equiv \alpha_{4} a^{3}+\beta_{4} a^{2} b \quad\left(\bmod J^{4}\right) .
\end{aligned}
$$

Hence $\alpha_{2}=\beta_{2}=\alpha_{3}=\beta_{3}=\alpha_{4}=\beta_{4}=0$; in particular, $c a, c b, c^{2} \in J^{3}$. Thus

$$
0 \equiv b\left(c^{2}\right) \equiv(b c) c \equiv \alpha_{1} a^{2} c+\beta_{1} a b c \equiv \alpha_{1} \beta_{1} a^{3}+\beta_{1}^{2} a^{2} b\left(\bmod J^{4}\right),
$$

and we obtain $\beta_{1}=0$. Thus $0 \equiv b(c b) \equiv(b c) b \equiv \alpha_{1} a^{2} b \quad\left(\bmod J^{4}\right)$. Therefore $\alpha_{1}=0$; in particular, $b c \in J^{3}$. Thus $[a, c],[b, c] \in J^{3}$ and $K \subset$ $F\{[a, b],[a, c],[b, c]\}+J^{3} \subset F[a, b]+J^{3} ;$ in particular, $\operatorname{dim} K+J^{3} / J^{3} \leq 1$. Thus $\operatorname{dim} A / J^{3} \leq 5$ by (1.2), a contradiction.

\section{The CASE (1.5)}

In this section we assume hypothesis and notation from $\S 1$. In addition, we assume that (1.5) holds. Since $J=F\{a, b, c\}+J^{2}$ we have $J^{2}=$ $F\left\{a^{2}, a b, a c, b a, b^{2}, b c, c a, c b, c^{2}\right\}+J^{3}$. Since $\operatorname{dim} J^{2} / J^{3}=3$ we must have $J^{2}=F\left\{a^{2}, a b, d\right\}+J^{3}$ for some element $d \in\left\{a c, b a, b^{2}, b c, c a, c b\right.$, $\left.c^{2}\right\}$. Since $J^{2}=K+J^{4}$ we obtain

$$
\begin{aligned}
J^{2}=F\{[a, b],[a, c],[a, a b],[a, d],[b, c], \\
{\left.\left[b, a^{2}\right],[b, a b],[b, d],\left[c, a^{2}\right],[c, a b],[c, d]\right\}+J^{4} . }
\end{aligned}
$$

We choose elements $\alpha_{i}, \beta_{i}, \gamma_{i} \in F \quad(i=1,2, \ldots, 7)$ such that

$$
\begin{array}{rlll}
a c \equiv \alpha_{1} a^{2}+\beta_{1} a b+\gamma_{1} d & \left(\bmod J^{3}\right), & b a \equiv \alpha_{2} a^{2}+\beta_{2} a b+\gamma_{2} d & \left(\bmod J^{3}\right), \\
b^{2} \equiv \alpha_{3} a^{2}+\beta_{3} a b+\gamma_{3} d & \left(\bmod J^{3}\right), & b c \equiv \alpha_{4} a^{2}+\beta_{4} a b+\gamma_{4} d & \left(\bmod J^{3}\right), \\
c a \equiv \alpha_{5} a^{2}+\beta_{5} a b+\gamma_{5} d & \left(\bmod J^{3}\right), & c b \equiv \alpha_{6} a^{2}+\beta_{6} a b+\gamma_{6} d & \left(\bmod J^{3}\right), \\
c^{2} \equiv \alpha_{7} a^{2}+\beta_{7} a b+\gamma_{7} d & \left(\bmod J^{3}\right) . & &
\end{array}
$$

(4.1) Lemma. We may assume that $d=a c$ or $d=b a$.

Proof. Case 1. $d=a c$. In this case there is nothing to prove. 
Case 2. $d=b a$. In this case there is nothing to prove either.

Case 3. $d=b^{2}$. In this case we may assume that $b a \in F a^{2}+F a b+J^{3}$; for otherwise we are in Case 2. Similarly, we may assume that $b a \in F b^{2}+F a b+J^{3}$; for otherwise we interchange $a$ and $b$ and are in Case 2 again. Hence $b a \in$ $F a b+J^{3}$, and we may write $b a \equiv \alpha a b\left(\bmod J^{3}\right)$ for some element $\alpha \in F$. Now we set $b^{\prime}:=a+b$. Then we have

$$
a b^{\prime}=a^{2}+a b, \quad\left(b^{\prime}\right)^{2} \equiv a^{2}+(1+\alpha) a b+b^{2} \quad\left(\bmod J^{3}\right) ;
$$

in particular, $J=F\left\{a, b^{\prime}, c\right\}+J^{2}$ and $J^{2}=F\left\{a^{2}, a b^{\prime},\left(b^{\prime}\right)^{2}\right\}+J^{3}$. Hence we may similarly assume that $b^{\prime} a \in F a b^{\prime}+J^{3}$. We write $b^{\prime} a \equiv \beta a b^{\prime}\left(\bmod J^{3}\right)$ with some element $\beta \in F$. Then

$$
\beta a^{2}+\beta a b \equiv \beta a b^{\prime} \equiv b^{\prime} a \equiv(a+b) a \equiv a^{2}+b a \equiv a^{2}+\alpha a b \quad\left(\bmod J^{3}\right) .
$$

Since $a^{2}+J^{3}$ and $a b+J^{3}$ are linearly independent this means that $\alpha=\beta=1$; in particular, $[a, b] \in J^{3}$, and $J^{2}=F[a, c]+F[b, c]+J^{3}$ contradicting the fact that $\operatorname{dim} J^{2} / J^{3}=3$.

Case 4. $d=b c$. In this case we may assume that $a c, b a, b^{2} \in F a^{2}+F a b+J^{3}$; for otherwise we are in Cases 1, 2 or 3 again. Then we replace $c$ by $c-\alpha_{1} a-\beta_{1} b$ and may therefore assume that $0=\alpha_{1}=\beta_{1}$. Moreover, we may assume that

$$
\begin{aligned}
J^{2} & \neq(a+\xi b) J+J^{3}=F\{(a+\xi b) a,(a+\xi b) b,(a+\xi b) c\}+J^{3} \\
& =F\left\{\left(1+\alpha_{2} \xi\right) a^{2}+\beta_{2} \xi a b, \alpha_{3} \xi a^{2}+\left(1+\beta_{3} \xi\right) a b, \xi b c\right\}+J^{3}
\end{aligned}
$$

for $\xi \in F$; for otherwise we replace $a$ by $a+\xi b$ and are in Case 1 . Since $a^{2}+J^{3}, a b+J^{3}, b c+J^{3}$ form a basis of $J^{2} / J^{3}$ this implies that

$$
0=\left|\begin{array}{lll}
1+\alpha_{2} \xi & \beta_{2} \xi & 0 \\
\alpha_{3} \xi & 1+\beta_{3} \xi & 0 \\
0 & 0 & \xi
\end{array}\right|=\xi+\left(\alpha_{2}+\beta_{3}\right) \xi^{2}+\left(\alpha_{2} \beta_{3}-\alpha_{3} \beta_{2}\right) \xi^{3}
$$

for $\xi \in F$. Since $F$ is infinite this is impossible.

Case 5. $d=c a$, i.e., $\alpha_{5}=\beta_{5}=0, \gamma_{5}=1$. We may assume that $\gamma_{i}=0$ for $i=1,2,3,4$; for otherwise we are in Cases $1,2,3,4$, respectively. Then we replace $c$ by $c-\alpha_{1} a-\beta_{1} b$ and may therefore assume that $0=\alpha_{1}=\beta_{1}$. Moreover, $\beta_{2}=0$; for otherwise we are in Case 1. Similarly, we may assume that $\alpha_{3}=0$; for otherwise we interchange $a$ and $b$ and are then in Case 4 for the opposite algebra of $A$. Now we replace $b$ by $b-\gamma_{6} a$ and may then assume that $\gamma_{6}=0$. Furthermore, we may assume that $\alpha_{7}=0$; for otherwise we replace $(a, b, c)$ by $(b, c, a)$ and are then in Case 4 again. Finally, we may assume that $\beta_{7}=0$; for otherwise we interchange $b$ and $c$ and are then in Case 3 for the opposite algebra of $A$. As in Case 4, we may assume

$$
\begin{aligned}
J^{2} & \neq F\{(\xi a+\eta b+c) a,(\xi a+\eta b+c) b,(\xi a+\eta b+c) c\}+J^{3} \\
= & F\left\{\left(\xi+\alpha_{2} \eta\right) a^{2}+c a, \alpha_{6} a^{2}+\left(\xi+\beta_{3} \eta+\beta_{6}\right) a b,\right. \\
& \left.\alpha_{4} \eta a^{2}+\beta_{4} \eta a b+\gamma_{7} c a\right\}+J^{3}
\end{aligned}
$$

for $\xi, \eta \in F$. Since $a^{2}+J^{3}, a b+J^{3}, c a+J^{3}$ form a basis of $J^{2} / J^{3}$ we may compute the corresponding determinant and obtain

$$
\begin{aligned}
0= & \gamma_{7} \xi^{2}+\left(\beta_{3} \gamma_{7}+\alpha_{2} \gamma_{7}-\alpha_{4}\right) \xi \eta+\beta_{6} \gamma_{7} \xi+\left(\alpha_{2} \beta_{3} \gamma_{7}-\alpha_{4} \beta_{3}\right) \eta^{2} \\
& +\left(\alpha_{2} \beta_{6} \gamma_{7}+\alpha_{6} \beta_{4}-\alpha_{4} \beta_{6}\right) \eta
\end{aligned}
$$


for $\xi, \eta \in F$. Since $F$ is infinite this implies that all coefficients on the righthand side vanish; in particular, $0=\gamma_{7}=\alpha_{4}$. Then, similarly, we may assume that

$$
\begin{aligned}
J^{2} & \neq F\{a(a+\eta b+c), b(a+\eta b+c), c(a+\eta b+c)\}+J^{3} \\
& =F\left\{a^{2}+\eta a b, \alpha_{2} a^{2}+\left(\beta_{3} \eta+\beta_{4}\right) a b, \alpha_{6} \eta a^{2}+\beta_{6} \eta a b+c a\right\}+J^{3}
\end{aligned}
$$

for $\eta \in F$. Computing the corresponding determinant we obtain $0=$ $\left(\beta_{3}-\alpha_{2}\right) \eta+\beta_{4}$ for $\eta \in F$. As before this implies that $\beta_{3}=\alpha_{2}$ and $\beta_{4}=0$. Finally, we may assume that

$$
\begin{aligned}
& J^{2} \neq F\left\{(\xi a+b+c)^{2},(\xi a+b+c) a, a(\xi a+b+c)\right\}+J^{3} \\
&= F\left\{\left(\xi^{2}+\alpha_{2} \xi+\alpha_{6}\right) a^{2}+\left(\xi+\alpha_{2}+\beta_{6}\right) a b+\xi c a,\right. \\
&\left.\left(\xi+\alpha_{2}\right) a^{2}+c a, \xi a^{2}+a b\right\}+J^{3}
\end{aligned}
$$

for $\xi \in F$; for otherwise we replace $(a, b)$ by $(\xi a+b+c, a)$ and are in Case 2 again. Computing the corresponding determinant we obtain $0=\xi^{2}+$ $\left(\alpha_{2}+\beta_{6}\right) \xi-\alpha_{6}$ for $\xi \in F$ which is impossible.

Case 6. $d=c b$, i.e. $\alpha_{6}=\beta_{6}=0, \gamma_{6}=1$. We may assume that $\gamma_{i}=0$ for $i=1,2, \ldots, 5$; for otherwise we are in Cases $1,2, \ldots, 5$, respectively. Then we replace $c$ by $c-\alpha_{1} a-\beta_{1} b$ and may therefore assume that $0=\alpha_{1}=\beta_{1}$. We may also assume that $\beta_{2}=0$; for otherwise we are in Case 4 for the opposite algebra of $A$. Similarly, we may assume that $\alpha_{3}=0$; for otherwise we interchange $a$ and $b$ and are then in Case 1. As in the previous cases we may assume

$$
\begin{aligned}
J^{2} & \neq F\{(\xi a+\eta b+c) a,(\xi a+\eta b+c) b,(\xi a+\eta b+c) c\}+J^{3} \\
& =F\left\{\left(\xi+\alpha_{2} \eta+\alpha_{5}\right) a^{2}+\beta_{5} a b,\left(\xi+\beta_{3} \eta\right) a b+c b,\right. \\
& \left.\left(\alpha_{4} \eta+\alpha_{7}\right) a^{2}+\left(\beta_{4} \eta+\beta_{7}\right) a b+\gamma_{7} c b\right\}+J^{3}
\end{aligned}
$$

for $\xi, \eta \in F$. We work out the corresponding determinant and obtain

$$
\begin{aligned}
0= & \gamma_{7} \xi^{2}+\left(\beta_{3} \gamma_{7}+\alpha_{2} \gamma_{7}-\beta_{4}\right) \xi \eta+\left(\alpha_{5} \gamma_{7}-\beta_{7}\right) \xi+\left(\alpha_{2} \beta_{3} \gamma_{7}-\alpha_{2} \beta_{4}\right) \eta^{2} \\
& +\left(\alpha_{5} \beta_{3} \gamma_{7}-\alpha_{2} \beta_{7}-\alpha_{5} \beta_{4}+\alpha_{4} \beta_{5}\right) \eta+\left(\alpha_{7} \beta_{5}-\alpha_{5} \beta_{7}\right)
\end{aligned}
$$

for $\xi, \eta \in F$. Therefore all coefficients on the right-hand side vanish; in particular, $0=\gamma_{7}=\beta_{4}=\beta_{7}$. Similarly, we have

$$
\begin{aligned}
J^{2} & \neq F\{a(\xi a+b+c), b(\xi a+b+c), c(\xi a+b+c)\}+J^{3} \\
& =F\left\{\xi a^{2}+a b,\left(\alpha_{2} \xi+\alpha_{4}\right) a^{2}+\beta_{3} a b,\left(\alpha_{5} \xi+\alpha_{7}\right) a^{2}+\beta_{5} \xi a b+c b\right\}+J^{3}
\end{aligned}
$$

for $\xi \in F$. Computing the corresponding determinant we obtain $0=$ $\left(\beta_{3}-\alpha_{2}\right) \xi-\alpha_{4}$ for $\xi \in F$ which again implies that $\beta_{3}=\alpha_{2}$ and $\alpha_{4}=0$. We may also assume that

$$
\begin{aligned}
& J^{2} \neq F\left\{(\xi a+\eta b+c)^{2},(\xi a+\eta b+c) a, a(\xi a+\eta b+c)\right\}+J^{3} \\
&= F\left\{\left(\xi^{2}+\alpha_{2} \xi \eta+\alpha_{5} \xi+\alpha_{7}\right) a^{2}+\left(\xi \eta+\beta_{5} \xi+\alpha_{2} \eta^{2}\right) a b+\eta c b,\right. \\
&\left.\left(\xi+\alpha_{2} \eta+\alpha_{5}\right) a^{2}+\beta_{5} a b, \xi a^{2}+\eta a b\right\}+J^{3}
\end{aligned}
$$

for $\xi, \eta \in F$; for otherwise we replace $(a, b, c)$ by $(\xi a+\eta b+c, a, b)$ and are then in Case 2 again. Working out the corresponding determinant we obtain $0=\xi \eta^{2}+\alpha_{2} \eta^{3}-\beta_{5} \xi \eta+\alpha_{5} \eta^{2}$ for $\xi, \eta \in F$ which is impossible. 
Case 7. $d=c^{2}$. In this case we may assume that $a c, b a, b^{2}, b c, c a, c b \in$ $F a^{2}+F a b+J^{3}$; for otherwise we are in Cases $1,2, \ldots, 6$, respectively. Then $J^{2}=F\{[a, b],[a, c],[b, c]\}+J^{3} \subset F a^{2}+F a b+J^{3}$; in particular, $\operatorname{dim} J^{2} / J^{3} \leq 2$ contradicting (1.5).

(4.2) Lemma. We may assume that $d=a c$.

Proof. We assume the contrary. Then we may assume that $d=b a$, by (4.1); in particular, $\alpha_{2}=\beta_{2}=0, \gamma_{2}=1$. We have $\gamma_{1}=0$. After replacing $c$ by $c-\alpha_{1} a-\beta_{1} b$ we may even assume $0=\alpha_{1}=\beta_{1}$. Similarly, we may assume $\beta_{5}=0$. Moreover, after replacing $b$ by $b-\gamma_{3} a$ we may also assume that $\gamma_{3}=0$. We then have

$$
\begin{aligned}
J^{2} & \neq(\xi a+\eta b+\zeta c) J+J^{3} \\
= & F\{(\xi a+\eta b+\zeta c) a,(\xi a+\eta b+\zeta c) b,(\xi a+\eta b+\zeta c) c\}+J^{3} \\
= & F\left\{\left(\xi+\alpha_{5} \zeta\right) a^{2}+\left(\eta+\gamma_{5} \zeta\right) b a,\left(\alpha_{3} \eta+\alpha_{6} \zeta\right) a^{2}+\left(\xi+\beta_{3} \eta+\beta_{6} \zeta\right) a b\right. \\
& \left.\quad+\gamma_{6} \zeta b a,\left(\alpha_{4} \eta+\alpha_{7} \zeta\right) a^{2}+\left(\beta_{4} \eta+\beta_{7} \zeta\right) a b+\left(\gamma_{4} \eta+\gamma_{7} \zeta\right) b a\right\}+J^{3}
\end{aligned}
$$

for $\xi, \eta, \zeta \in F$. Since $a^{2}+J^{3}, a b+J^{3}, b a+J^{3}$ form a basis of $J^{2} / J^{3}$ this implies that

$$
\begin{aligned}
0= & \left|\begin{array}{lll}
\xi+\alpha_{5} \zeta & 0 & \eta+\gamma_{5} \zeta \\
\alpha_{3} \eta+\alpha_{6} \zeta & \xi+\beta_{3} \eta+\beta_{6} \zeta & \gamma_{6} \zeta \\
\alpha_{4} \eta+\alpha_{7} \zeta & \beta_{4} \eta+\beta_{7} \zeta & \gamma_{4} \eta+\gamma_{7} \zeta
\end{array}\right| \\
= & \gamma_{4} \xi^{2} \eta+\gamma_{7} \xi^{2} \zeta+\left(\beta_{3} \gamma_{4}-\alpha_{4}\right) \xi \eta^{2} \\
& +\left(\beta_{3} \gamma_{7}+\beta_{6} \gamma_{4}+\alpha_{5} \gamma_{4}-\beta_{4} \gamma_{6}-\alpha_{7}-\alpha_{4} \gamma_{5}\right) \xi \eta \zeta \\
& +\left(\beta_{6} \gamma_{7}+\alpha_{5} \gamma_{7}-\beta_{7} \gamma_{6}-\alpha_{7} \gamma_{5}\right) \xi \zeta^{2}+\left(\alpha_{3} \beta_{4}-\alpha_{4} \beta_{3}\right) \eta^{3} \\
& +\left(\alpha_{5} \beta_{3} \gamma_{4}+\alpha_{3} \beta_{7}+\alpha_{6} \beta_{4}+\alpha_{3} \beta_{4} \gamma_{5}-\alpha_{4} \beta_{6}-\alpha_{7} \beta_{3}-\alpha_{4} \beta_{3} \gamma_{5}\right) \eta^{2} \zeta \\
& +\left(\alpha_{5} \beta_{3} \gamma_{7}+\alpha_{5} \beta_{6} \gamma_{4}-\alpha_{5} \beta_{4} \gamma_{6}+\alpha_{6} \beta_{7}+\alpha_{3} \beta_{7} \gamma_{5}+\alpha_{6} \beta_{4} \gamma_{5}\right. \\
& +\left(\alpha_{5} \beta_{6} \gamma_{7}-\alpha_{5} \beta_{7} \gamma_{6}+\alpha_{6} \beta_{7} \gamma_{5}-\alpha_{7} \beta_{6} \gamma_{5}\right) \zeta^{3}
\end{aligned}
$$

for $\xi, \eta, \zeta \in F$. Since $F$ is infinite this implies that all coefficients on the right-hand side have to vanish; in particular, $0=\gamma_{4}=\gamma_{7}=\alpha_{4}=\alpha_{3} \beta_{4}$ and $\alpha_{7}=-\beta_{4} \gamma_{6}$. Then, similarly, we have

$$
\begin{aligned}
J^{2} \neq & F\{a(\xi a+\eta b+\zeta c), b(\xi a+\eta b+\zeta c), c(\xi a+\eta b+\zeta c)\}+J^{3} \\
= & F\left\{\xi a^{2}+\eta a b, \alpha_{3} \eta a^{2}+\left(\beta_{3} \eta+\beta_{4} \zeta\right) a b+\xi b a,\right. \\
& \left.\left(\alpha_{5} \xi+\alpha_{6} \eta+\alpha_{7} \zeta\right) a^{2}+\left(\beta_{6} \eta+\beta_{7} \zeta\right) a b+\left(\gamma_{5} \xi+\gamma_{6} \eta\right) b a\right\}+J^{3}
\end{aligned}
$$

for $\xi, \eta, \zeta \in F$. As before, we work out the corresponding determinant and obtain

$$
0=\left(\beta_{3} \gamma_{5}-\beta_{6}+\alpha_{5}\right) \xi^{2} \eta+\left(\beta_{4} \gamma_{5}-\beta_{7}\right) \xi^{2} \zeta+\left(\beta_{3} \gamma_{6}-\alpha_{3} \gamma_{5}+\alpha_{6}\right) \xi \eta^{2}-\alpha_{3} \gamma_{6} \eta^{3}
$$

for $\xi, \eta, \zeta \in F$. Again, this implies that $\beta_{6}=\beta_{3} \gamma_{5}+\alpha_{5}, \beta_{7}=\beta_{4} \gamma_{5}, \alpha_{6}=$ $\alpha_{3} \gamma_{5}-\beta_{3} \gamma_{6}, 0=\alpha_{3} \gamma_{6}$. On the other hand,

$$
\begin{aligned}
J^{2}=F\{[a, b],[a, c],[b, c]\} & +J^{3} \\
=F\left\{a b-b a, \alpha_{5} a^{2}+\gamma_{5} b a,\right. & \left(\beta_{3} \gamma_{6}-\alpha_{3} \gamma_{5}\right) a^{2} \\
& \left.+\left(\beta_{4}-\alpha_{5}-\beta_{3} \gamma_{5}\right) a b-\gamma_{6} b a\right\}+J^{3} .
\end{aligned}
$$


Since $a^{2}+J^{3}, a b+J^{3}$ and $b a+J^{3}$ form a basis of $J^{2} / J^{3}$ a computation of the corresponding determinant yields

$$
0 \neq \alpha_{5} \gamma_{6}+\beta_{3} \gamma_{5} \gamma_{6}-\alpha_{3} \gamma_{5}^{2}-\alpha_{5} \beta_{4}+\alpha_{5}^{2}+\alpha_{5} \beta_{3} \gamma_{5} .
$$

Moreover, since $J^{2}=F\left\{a^{2}, a b, b a\right\}+J^{3}$, Lemma $\mathrm{E}$ in [6] implies that $J^{3}=F\left\{a^{3}, a^{2} b, a b a, b a^{2}, b a b, b^{2} a\right\}+J^{4}=F\left\{a^{3}, a^{2} b, a b a, b a^{2}, b a b\right\}+J^{4}$.

Now we distinguish two cases.

Case 1. $\alpha_{5} \neq 0$. In this case we replace $a$ by $\alpha_{5} a$ and may then assume that $\alpha_{5}=1$. Thus

$$
\begin{aligned}
& 0 \equiv a(c a)-(a c) a \equiv a^{3}+\gamma_{5} a b a \quad\left(\bmod J^{4}\right), \\
& 0 \equiv b(c a)-(b c) a \equiv\left(\beta_{3} \gamma_{5}-\alpha_{3} \gamma_{5}^{2}-\beta_{4}\right) a b a+b a^{2} \quad\left(\bmod J^{4}\right) .
\end{aligned}
$$

Now we distinguish two more cases.

Case 1.1. $\beta_{4} \neq 0$. In this case we have $\alpha_{3}=0$ since $0=\alpha_{3} \beta_{4}$. Moreover,

$$
\begin{aligned}
& 0 \equiv\left(b^{2}\right) c-b(b c) \equiv \beta_{3} \beta_{4} a^{2} b-\beta_{4} b a b \quad\left(\bmod J^{4}\right), \\
& 0 \equiv a\left(c^{2}\right)-(a c) c \equiv \beta_{4} \gamma_{5} a^{2} b+\beta_{4} \gamma_{5} \gamma_{6} a b a \quad\left(\bmod J^{4}\right) ;
\end{aligned}
$$

in particular, $J^{3}=F a^{2} b+F a b a+J^{4}$. Hence $a^{2} b+J^{4}$ and $a b a+J^{4}$ are linearly independent. Then $\gamma_{5}=0$, and we obtain the contradiction

$$
0 \equiv a(c b)-(a c) b \equiv a^{2} b+\gamma_{6} a b a \quad\left(\bmod J^{4}\right) .
$$

Case 1.2. $\beta_{4}=0$. Here we have to distinguish two more cases.

Case 1.2.1. $\beta_{3} \neq 0$. In this case we replace $b$ by $\beta_{3}^{-1} b$ and may then assume that $\beta_{3}=1$. Then

$$
\begin{aligned}
& 0 \equiv\left(b^{2}\right) b-b\left(b^{2}\right) \equiv\left(1+\alpha_{3}\right) a^{2} b-\alpha_{3}^{2} \gamma_{5}^{2} a b a-b a b \quad\left(\bmod J^{4}\right), \\
& 0 \equiv a(c b)-(a c) b \equiv\left(1+\gamma_{5}\right) a^{2} b+\left(\gamma_{5} \gamma_{6}-\alpha_{3} \gamma_{5}^{2}+\gamma_{6}\right) a b a \quad\left(\bmod J^{4}\right) ;
\end{aligned}
$$

in particular, $J^{3}=F a^{2} b+F a b a+J^{4}$. Thus $a^{2} b+J^{4}$ and $a b a+J^{4}$ are linearly independent. Then $\gamma_{5}=-1$ and $\alpha_{3}=0$. But now we obtain the contradiction

$$
\alpha_{5} \gamma_{6}+\beta_{3} \gamma_{5} \gamma_{6}-\alpha_{3} \gamma_{5}^{2}-\alpha_{5} \beta_{4}+\alpha_{5}^{2}+\alpha_{5} \beta_{3} \gamma_{5}=0 \text {. }
$$

Case 1.2.2. $\beta_{3}=0$. Here we have

$$
\begin{aligned}
& 0 \equiv a(c b)-(a c) b \equiv a^{2} b+\left(\gamma_{6}-\alpha_{3} \gamma_{5}^{2}\right) a b a \quad\left(\bmod J^{4}\right), \\
& 0 \equiv b(c b)-(b c) b \equiv \alpha_{3}^{2} \gamma_{5}^{3} a b a+b a b \quad\left(\bmod J^{4}\right) ;
\end{aligned}
$$

in particular, $J^{3}=F a b a+J^{4}$, a contradiction.

Case 2. $\alpha_{5}=0$. Then

$$
0 \neq \alpha_{5} \gamma_{6}+\beta_{3} \gamma_{5} \gamma_{6}-\alpha_{3} \gamma_{5}^{2}-\alpha_{5} \beta_{4}+\alpha_{5}^{2}+\alpha_{5} \beta_{3} \gamma_{5}=\beta_{3} \gamma_{5} \gamma_{6}-\alpha_{3} \gamma_{5}^{2}
$$

in particular, $\gamma_{5} \neq 0$. Now we replace $b$ by $\gamma_{5} b$ and may therefore assume that $\gamma_{5}=1$. Hence

$$
0 \equiv a(c a)-(a c) a \equiv a b a \quad\left(\bmod J^{4}\right) .
$$

We distinguish two more cases. 
Case 2.1. $\alpha_{3} \neq 0$. In this case $\beta_{4}=\gamma_{6}=0$ since $0=\alpha_{3} \beta_{4}=\alpha_{3} \gamma_{6}$. We now replace $a$ by $\sqrt{\alpha_{3}} a$ and may therefore assume that $\alpha_{3}=1$. Then

$$
\begin{aligned}
& 0 \equiv b(c a)-(b c) a \equiv a^{3} \quad\left(\bmod J^{4}\right), \\
& 0 \equiv b(c b)-(b c) b \equiv b a^{2}+\beta_{3} b a b \quad\left(\bmod J^{4}\right), \\
& 0 \equiv a(c b)-(a c) b \equiv \beta_{3} a^{2} b \quad\left(\bmod J^{4}\right), \\
& 0 \equiv\left(b^{2}\right) b-b\left(b^{2}\right) \equiv a^{2} b \quad\left(\bmod J^{4}\right) ;
\end{aligned}
$$

in particular, $J^{3}=F b a b+J^{4}$, a contradiction.

Case 2.2. $\alpha_{3}=0$. In this case we have $0 \neq \beta_{3} \gamma_{6}-\alpha_{3}=\beta_{3} \gamma_{6}$, i.e. $\beta_{3} \neq 0 \neq \gamma_{6}$. We now replace $a$ by $\beta_{3} a$ and may then assume that $\beta_{3}=1$. We compute

$$
\begin{aligned}
& 0 \equiv a(c b)-(a c) b \equiv a^{2} b-\gamma_{6} a^{3} \quad\left(\bmod J^{4}\right), \\
& 0 \equiv\left(b^{2}\right) b-b\left(b^{2}\right) \equiv \gamma_{6} a^{3}-b a b \quad\left(\bmod J^{4}\right), \\
& 0 \equiv(b c) c-b\left(c^{2}\right) \equiv\left(\beta_{4}^{2} \gamma_{6}-\beta_{4} \gamma_{6}\right) a^{3}+\beta_{4} \gamma_{6} b a^{2} \quad\left(\bmod J^{4}\right) ;
\end{aligned}
$$

in particular, $J^{3}=F a^{3}+F b a^{2}+J^{4}$. Thus $a^{3}+J^{4}$ and $b a^{2}+J^{4}$ are linearly independent. Then $\beta_{4}=0$ since $\gamma_{6} \neq 0$. But now we obtain the contradiction

$$
0 \equiv b(c b)-(b c) b \equiv \gamma_{6} a^{3}-\gamma_{6} b a^{2} \quad\left(\bmod J^{4}\right) \text {. }
$$

In the remainder of this paper we may and will assume that $J^{2}=F\left\{a^{2}, a b, a c\right\}$ $+J^{3}$. Then $J^{3}=F\left\{a^{3}, a^{2} b, a^{2} c\right\}+J^{4}$ and $J^{4}=F\left\{a^{4}, a^{3} b, a^{3} c\right\}+J^{5}$ by Lemma $E$ in [6]; in particular, $\operatorname{dim} J^{3} / J^{4} \in\{2,3\}$. Since $J^{4} \neq J^{5}$ we have $a^{3} \notin J^{4}$.

(4.3) Lemma. The elements $a, b, c$ can be chosen such that one of the following holds:

(4.4) $0=\alpha_{2}=\beta_{2}=\alpha_{5}, \gamma_{2}=1, \alpha_{6}=\alpha_{4}-1 ; \beta_{5}+\gamma_{5} \neq 1$;

(4.5) $0=\alpha_{2}=\beta_{2}, \gamma_{2}=\alpha_{5}=1, \gamma_{5}=1-\beta_{5}, \beta_{6}-\beta_{4}+\gamma_{6}-\gamma_{4} \neq 0$;

(4.6) $0=\alpha_{2}=\gamma_{2}=\alpha_{5}=\beta_{5}, \gamma_{5}=\beta_{2} \neq 1, \alpha_{4}=1 \neq \alpha_{6}$.

Proof. We distinguish between two cases.

Case 1. $\gamma_{2} \neq 0$. In this case we replace $c$ by $\alpha_{2} a+\beta_{2} b+\gamma_{2} c$ and may therefore assume that $0=\alpha_{2}=\beta_{2}$ and $\gamma_{2}=1$. Now we distinguish two more cases.

Case 1.1. $\beta_{5}+\gamma_{5} \neq 1$. In this case we set $\xi:=\alpha_{5} /\left(\beta_{5}+\gamma_{5}-1\right)$ and replace $b$ by $b+\xi a$ and $c$ by $c+\xi a$. Then we have $\alpha_{5}=0$. Hence

$$
\begin{aligned}
J^{2}=F\{[a, b],[a, c],[b, c]\}+J^{3} & \\
=F\left\{a b-a c, \beta_{5} a b+\left(\gamma_{5}-1\right) a c,\right. & \left(\alpha_{4}-\alpha_{6}\right) a^{2} \\
& \left.+\left(\beta_{4}-\beta_{6}\right) a b+\left(\gamma_{4}-\gamma_{6}\right) a c\right\}+J^{3} ;
\end{aligned}
$$

in particular, $\alpha_{4} \neq \alpha_{6}$. Now we replace $a$ by $\left(\alpha_{4}-\alpha_{6}\right)^{1 / 2} a$ and may then assume that $\alpha_{6}=\alpha_{4}-1$.

Case 1.2. $\beta_{5}+\gamma_{5}=1$. In this case we have

$$
\begin{aligned}
J^{2}= & F\{[a, b],[a, c],[b, c]\}+ \\
=F\left\{a b-a c, \alpha_{5} a^{2}+\beta_{5} a b-\right. & \beta_{5} a c,\left(\alpha_{4}-\alpha_{6}\right) a^{2} \\
& \left.+\left(\beta_{4}-\beta_{6}\right) a b+\left(\gamma_{4}-\gamma_{6}\right) a c\right\}+J^{3} .
\end{aligned}
$$


Since $a^{2}+J^{3}, a b+J^{3}, a c+J^{3}$ form a basis of $J^{2} / J^{3}$ we work out the corresponding determinant and obtain $0 \neq \alpha_{5}\left(\beta_{6}-\beta_{4}+\gamma_{6}-\gamma_{4}\right)$, so $\beta_{6}-\beta_{4}+$ $\gamma_{6}-\gamma_{4} \neq 0 \neq \alpha_{5}$. Then we replace $a$ by $\alpha_{5} a$ and may therefore assume that $\alpha_{5}=1$.

Case 2. $\gamma_{2}=0$. In this case we may assume that $\beta_{5}=0$; for otherwise we interchange $b$ and $c$ and are then in Case 1 again. Similarly, we may assume that $\gamma_{5}=\beta_{2}$; otherwise we replace $b$ by $b+c$ and are then in Case 1 again. Hence

$$
\begin{aligned}
J^{2}=F\{[a, b],[a, c],[b, c]\} & +J^{3} \\
=F\left\{\alpha_{2} a^{2}+\left(\beta_{2}-1\right) a b, \alpha_{5} a^{2}\right. & +\left(\beta_{2}-1\right) a c,\left(\alpha_{4}-\alpha_{6}\right) a^{2} \\
& \left.+\left(\beta_{4}-\beta_{6}\right) a b+\left(\gamma_{4}-\gamma_{6}\right) a c\right\}+J^{3} .
\end{aligned}
$$

Since $\operatorname{dim} J^{2} / J^{3}=3$ this implies that $\beta_{2} \neq 1$. Now we replace $b$ by $b+$ $\alpha_{2}\left(\beta_{2}-1\right)^{-1} a$ and $c$ by $c+\alpha_{5}\left(\beta_{2}-1\right)^{-1} a$ and may then assume that $0=\alpha_{2}=$ $\alpha_{5}$. In this situation we have $\alpha_{4} \neq 0$ or $\alpha_{6} \neq 0$. If necessary, we interchange $b$ and $c$ and may then assume that $\alpha_{4} \neq 0$. Finally we replace $b$ by $\alpha_{4}^{-1} b$ and may therefore assume that $\alpha_{4}=1$.

Now we treat the cases above separately.

(4.7) Lemma. The case (4.4) does not occur.

Proof. We assume the contrary and distinguish two cases.

Case $1 . \operatorname{dim} J^{3} / J^{4}=3$. In this case the elements $a^{3}+J^{4}, a^{2} b+J^{4}, a^{2} c+J^{4}$ form a basis of $J^{3} / J^{4}$. Since

$$
\begin{aligned}
0 \equiv & \left(b^{2}\right) a-b(b a) \equiv\left(\alpha_{3}-\alpha_{7}\right) a^{3}+\left(\beta_{5} \gamma_{3}-\beta_{7}\right) a^{2} b \\
& +\left(\beta_{3}+\gamma_{3} \gamma_{5}-\gamma_{7}\right) a^{2} c \quad\left(\bmod J^{4}\right)
\end{aligned}
$$

we conclude that $\alpha_{7}=\alpha_{3}, \beta_{7}=\beta_{5} \gamma_{3}$ and $\gamma_{7}=\beta_{3}+\gamma_{3} \gamma_{5}$. Similarly, using the fact that $0=(b c) a-b(c a)+c(b a)-(c b) a+J^{4}$ we obtain $\beta_{5}=-1$, so $\gamma_{5} \neq 2$. This also shows that $\gamma_{6}=\beta_{4}-\beta_{6}+\gamma_{4}$ and $0=\left(2-\gamma_{5}\right)\left(\beta_{4}-\beta_{6}\right)$. Since $\gamma_{5} \neq 2$ this implies that $\beta_{6}=\beta_{4}$ and $\gamma_{6}=\gamma_{4}$. Then, using the fact that $0=\left(b^{2}\right) b-b\left(b^{2}\right)+J^{4}$ and $0=(b c) b-b(c b)+J^{4}$ we see that $0=$ $\left(\alpha_{3}-\alpha_{4}+1\right)\left(\beta_{3}-\gamma_{3}\right)=\left(\alpha_{3}-\alpha_{4}+1\right)\left(\beta_{4}-\gamma_{4}\right)$. Now we distinguish two cases.

Case 1.1. $\alpha_{4} \neq \alpha_{3}+1$. Then $\gamma_{3}=\beta_{3}$ and $\gamma_{4}=\beta_{4}$. Moreover, the fact that $0=(b c) a-b(c a)+J^{4}$ implies that $0=\beta_{3} \gamma_{5}$. We distinguish two more cases.

Case 1.1.1. $\gamma_{5} \neq 0$, so $\beta_{3}=0$. In this case we use the fact that $0=(b c) b-$ $b(c b)+J^{4}$ to obtain $0=\gamma_{5}\left(1-\alpha_{4}\right)$, so $\alpha_{4}=1$. But this leads to a contradiction using the fact that $0=(b c) b-b(c b)+J^{4}$ again.

Case 1.1.2. $\gamma_{5}=0$. In this case we use the fact that $0=(b c) b-b(c b)+J^{4}$ to obtain $2 \alpha_{4}=1$; in particular, $\operatorname{char} F \neq 2$. Then we use the fact that $0=(b c) a-b(c a)+J^{4}$ to conclude that $\beta_{4}=0$, we use the fact that $0=\left(c^{2}\right) a-$ $c(c a)+J^{4}$ to see that $\beta_{3}=0$, and we use the fact that $0=(b c) c-b\left(c^{2}\right)+J^{4}$ to show that $\alpha_{4}=0$. But this contradicts the fact that $0=(b c) b-b(c b)+J^{4}$.

Case 1.2. $\alpha_{4}=\alpha_{3}+1$. In this case the fact that $0=(b c) a-b(c a)+J^{4}$ implies 
that $0=2 \alpha_{3}+1-\alpha_{3} \gamma_{5}$. Thus

$$
\begin{gathered}
J^{2}=F\left\{[a, b],[a, c],[b, c],[a, a b],[a, a c],\left[b, a^{2}\right],\right. \\
\left.[b, a b],[b, a c],\left[c, a^{2}\right],[c, a b],[c, a c]\right\}+J^{4} \\
\subset F\left\{[a, b],[a, c],[b, c], a^{2} b, a^{2} c\right\}+J^{4}
\end{gathered}
$$

as is easily checked. But this is a contradiction since $\operatorname{dim} J^{2} / J^{4}=6$.

Case 2. $\operatorname{dim} J^{3} / J^{4}=2$. Here we distinguish two more cases.

Case 2.1. $a^{2} b \in F a^{3}+J^{4}$. In this case we have $J^{3}=F\left\{a^{3}, a^{2} b, a^{2} c\right\}+J^{4}=$ $F\left\{a^{3}, a^{2} c\right\}+J^{4}$ and write $a^{2} b \equiv \delta a^{3}\left(\bmod J^{4}\right)$ with some element $\delta \in F$. Then $a^{3} c \equiv a^{2} b a \equiv \delta a^{4}\left(\bmod J^{5}\right)$, so $J^{4}=F\left\{a^{4}, a^{3} c\right\}+J^{5}=F a^{4}+J^{5}$. Since $J^{4} \neq J^{5}$ this implies that $\operatorname{dim} J^{4} / J^{5}=1$. By Lemma G in [6], $J^{3} \subset Z$; in particular, $a^{2} c \in Z$. Hence

$$
0 \equiv\left(a^{2} c\right) a-a\left(a^{2} c\right) \equiv a^{2}(c a)-a^{3} c \equiv\left(\beta_{5}+\gamma_{5}-1\right) \delta a^{3} \quad\left(\bmod J^{4}\right) .
$$

Since $\beta_{5}+\gamma_{5} \neq 1$ and $a^{3} \notin J^{4}$ we conclude that $\delta=0$. But now

$$
\begin{aligned}
0 \equiv & a^{2}(b c)-\left(a^{2} b\right) c \equiv \alpha_{4} a^{4} \quad\left(\bmod J^{5}\right) \\
0 \equiv & a^{2}\left(b^{2}\right)-\left(a^{2} b\right) b \equiv \alpha_{3} a^{4} \quad\left(\bmod J^{5}\right), \\
0 \equiv & \left(b^{2}\right) a-b(b a) \equiv\left(\alpha_{3}-\alpha_{7}\right) a^{3}+\left(\beta_{3}+\gamma_{3} \gamma_{5}-\gamma_{7}\right) a^{2} c \quad\left(\bmod J^{4}\right), \\
0 \equiv & (c b) a-c(b a) \equiv\left(\alpha_{4}-1-\alpha_{4} \beta_{5}-\alpha_{7} \gamma_{5}\right) a^{3} \\
& +\left(\beta_{6}+\gamma_{5} \gamma_{6}-\beta_{5} \gamma_{4}-\gamma_{5} \gamma_{7}\right) a^{2} c \quad\left(\bmod J^{4}\right) .
\end{aligned}
$$

This leads to the contradiction $0=\alpha_{4}=\alpha_{3}=\alpha_{7}=-1$.

Case 2.2. $a^{2} b \notin F a^{3}+J^{4}$. Since $a^{3} \notin J^{4}$ and $\operatorname{dim} J^{3} / J^{4}=2$ the elements $a^{3}+J^{4}$ and $a^{2} b+J^{4}$ form a basis of $J^{3} / J^{4}$ in this case. We write $a^{2} c \equiv$ $\delta a^{3}+\varepsilon a^{2} b\left(\bmod J^{4}\right)$ with elements $\delta, \varepsilon \in F$. Since $J^{4}=F a^{4}+F a^{3} b+J^{5}$ and $J^{4} \neq J^{5}$ we have $\operatorname{dim} J^{4} / J^{5} \in\{1,2\}$. Let us distinguish the corresponding cases.

Case 2.2.1. $\operatorname{dim} J^{4} / J^{5}=1$. In this case $J^{3} \subset Z$ by Lemma $G$ in [6]; in particular, $a^{2} b, a^{2} c \in Z$. Hence

$$
\begin{aligned}
0 & \equiv\left(a^{2} b\right) a-a\left(a^{2} b\right) \equiv a^{2}(b a)-a^{3} b \\
& \equiv a^{3} c-a^{3} b \equiv \delta a^{4}+(\varepsilon-1) a^{3} b \quad\left(\bmod J^{5}\right), \\
0 & \equiv\left(a^{2} c\right) a-a\left(a^{2} c\right) \equiv a^{2}(c a)-a^{3} c \equiv\left(\gamma_{5}-1\right) \delta a^{4}+\left(\beta_{5}+\gamma_{5} \varepsilon-\varepsilon\right) a^{3} b \\
& \equiv\left(\beta_{5}+\gamma_{5}-1\right) a^{3} b \quad\left(\bmod J^{5}\right) .
\end{aligned}
$$

Since $\beta_{5}+\gamma_{5} \neq 1$ this implies that $a^{3} b \in J^{5}$. Hence $J^{4}=F a^{4}+J^{5}$ and $\delta a^{4} \in J^{5}$. Since $\operatorname{dim} J^{4} / J^{5}=1$ we must have $\delta=0$. Therefore

$$
\begin{aligned}
0 \equiv & \left(b^{2}\right) a-b(b a) \equiv\left(\alpha_{3}-\alpha_{7}\right) a^{3} \\
& +\left(\beta_{5} \gamma_{3}-\beta_{7}+\beta_{3} \varepsilon+\gamma_{3} \gamma_{5} \varepsilon-\gamma_{7} \varepsilon\right) a^{2} b \quad\left(\bmod J^{4}\right)
\end{aligned}
$$

in particular, $\alpha_{7}=\alpha_{3}$. Similarly, using the fact that $0=(b c) a-b(c a)+c(b a)-$ $(c b) a+J^{4}$ we see that $\beta_{5}=-1$; in particular, $\gamma_{5} \neq 2$. Hence

$$
\begin{aligned}
& 0 \equiv a^{2}(c b)-\left(a^{2} c\right) b \equiv\left(\alpha_{4}-1-\alpha_{3} \varepsilon\right) a^{4}\left(\bmod J^{5}\right), \\
& 0 \equiv a^{2}\left(c^{2}\right)-\left(a^{2} c\right) c \equiv\left(\alpha_{3}-\alpha_{4} \varepsilon\right) a^{4}\left(\bmod J^{5}\right)
\end{aligned}
$$


Since $a^{4} \notin J^{5}$ this implies that $\alpha_{3}=\alpha_{4} \varepsilon$ and $\alpha_{4}-\alpha_{4} \varepsilon^{2}=1$; in particular, $\alpha_{4} \neq 0$ and $\varepsilon^{2} \neq 1$. But since $a^{2} c \in Z$ we have

$$
\begin{aligned}
0 & \equiv\left(a^{2} c\right) b-b\left(a^{2} c\right) \equiv\left(a^{2} c\right) b-(b a)(a c) \\
& \equiv\left(\varepsilon^{2}+1-\gamma_{5} \varepsilon\right) \alpha_{4} a^{4} \quad\left(\bmod J^{5}\right), \\
0 & \equiv\left(a^{2} c\right) b-b\left(a^{2} c\right) \equiv\left(2-\gamma_{5} \varepsilon\right) \alpha_{4} \varepsilon^{2} a^{4} \quad\left(\bmod J^{5}\right) .
\end{aligned}
$$

Hence $\gamma_{5} \varepsilon=2$ and $\varepsilon^{2}=1$, a contradiction.

Case 2.2.2. $\operatorname{dim} J^{4} / J^{5}=2$. In this case

$$
\begin{aligned}
0 \equiv & \left(a^{2} c\right) a-a^{2}(c a) \equiv\left(\delta+\delta \varepsilon-\gamma_{5} \delta\right) a^{4}+\left(\varepsilon^{2}-\beta_{5}-\gamma_{5} \varepsilon\right) a^{3} b \quad\left(\bmod J^{5}\right), \\
0 \equiv & \left(a^{2} c\right) b-a^{2}(c b) \equiv\left(\alpha_{3} \varepsilon+\gamma_{3} \delta \varepsilon-\alpha_{4}+1-\gamma_{6} \delta\right) a^{4} \\
& +\left(\delta+\beta_{3} \varepsilon+\gamma_{3} \varepsilon^{2}-\beta_{6}-\gamma_{6} \varepsilon\right) a^{3} b \quad\left(\bmod J^{5}\right), \\
0 \equiv & \left(a^{2} c\right) c-a^{2} c^{2} \equiv\left(\alpha_{4} \varepsilon+\gamma_{4} \delta \varepsilon-\alpha_{7}-\gamma_{7} \delta+\delta^{2}\right) a^{4} \\
& +\left(\delta \varepsilon+\beta_{4} \varepsilon+\gamma_{4} \varepsilon^{2}-\beta_{7}-\gamma_{7} \varepsilon\right) a^{3} b \quad\left(\bmod J^{5}\right) .
\end{aligned}
$$

Since $a^{4}+J^{5}$ and $a^{3} b+J^{5}$ form a basis of $J^{4} / J^{5}$ this implies that all coefficients on the right-hand side vanish; in particular, $0=\delta+\delta \varepsilon-\gamma_{5} \delta$. Assume that $\delta \neq 0$. Then $\varepsilon=\gamma_{5}-1$ and we obtain the contradiction $0=\varepsilon^{2}-\beta_{5}-\gamma_{5} \varepsilon=1-\beta_{5}-\gamma_{5}$. Hence we must have $\delta=0$. Therefore

$$
\begin{aligned}
0 \equiv & \left(b^{2}\right) a-b(b a) \equiv\left(\alpha_{3}-\alpha_{7}\right) a^{3} \\
& +\left(\beta_{5} \gamma_{3}-\beta_{7}+\beta_{3} \varepsilon+\gamma_{3} \gamma_{5} \varepsilon-\gamma_{7} \varepsilon\right) a^{2} b \quad\left(\bmod J^{4}\right) ;
\end{aligned}
$$

in particular, $\alpha_{7}=\alpha_{3}$. Similarly, using the fact that $0=(b c) a-b(c a)+c(b a)-$ $(c b) a+J^{4}$ we see that $\beta_{5}=-1$. Hence $\varepsilon^{2}-\gamma_{5} \varepsilon=-1$; in particular, $\varepsilon \neq 0$. Therefore $0=\alpha_{3} \varepsilon+\gamma_{3} \delta \varepsilon-\alpha_{4}+1-\gamma_{6} \delta=\alpha_{3} \varepsilon-\alpha_{4}+1$, and $\alpha_{4}=\alpha_{3} \varepsilon+1$. Hence $0=\alpha_{4} \varepsilon+\gamma_{4} \delta \varepsilon-\alpha_{7}-\gamma_{7} \delta+\delta^{2}=\alpha_{3} \varepsilon^{2}+\varepsilon-\alpha_{3}$; in particular, $\alpha_{3} \neq 0$ and $\varepsilon^{2} \neq 1$. But this leads to the contradiction

$$
\begin{aligned}
0 & \equiv b\left(a^{2} c\right)-(b a) a c \\
& \equiv-\varepsilon^{2} a^{4}+\left(\beta_{6} \gamma_{5} \varepsilon-\beta_{3} \varepsilon+\gamma_{5} \gamma_{6} \varepsilon^{2}-\gamma_{3} \varepsilon^{2}-\beta_{7} \gamma_{5}+\beta_{4}-\gamma_{5} \gamma_{7} \varepsilon+\gamma_{4} \varepsilon\right) a^{3} b
\end{aligned}
$$

$\left(\bmod J^{5}\right)$.

(4.8) Lemma. The case (4.5) does not occur.

Proof. We assume the contrary and distinguish two cases.

Case 1. $\operatorname{dim} J^{3} / J^{4}=3$. In this case we have

$$
\begin{aligned}
0 \equiv & (b c) a-b(c a) \\
\equiv & \left(\alpha_{4}+\gamma_{4}-1-\alpha_{6} \beta_{5}-\alpha_{7}+\alpha_{7} \beta_{5}\right) a^{3}+\left(\beta_{5} \gamma_{4}-\beta_{5}-\beta_{5} \beta_{6}-\beta_{7}+\beta_{5} \beta_{7}\right) a^{2} b \\
& +\left(\beta_{4}+\gamma_{4}-\beta_{5} \gamma_{4}-1+\beta_{5}-\beta_{5} \gamma_{6}-\gamma_{7}+\beta_{5} \gamma_{7}\right) a^{2} c\left(\bmod J^{4}\right) .
\end{aligned}
$$

Since $a^{3}+J^{4}, a^{2} b+J^{4}, a^{2} c+J^{4}$ form a basis of $J^{3} / J^{4}$ we obtain

$$
\begin{aligned}
& 0=\alpha_{4}+\gamma_{4}-1-\alpha_{6} \beta_{5}-\alpha_{7}+\alpha_{7} \beta_{5}, \\
& 0=\beta_{5} \gamma_{4}-\beta_{5}-\beta_{5} \beta_{6}-\beta_{7}+\beta_{5} \beta_{7}, \\
& 0=\beta_{4}+\gamma_{4}-\beta_{5} \gamma_{4}-1+\beta_{5}-\beta_{5} \gamma_{6}-\gamma_{7}+\beta_{5} \gamma_{7} .
\end{aligned}
$$


Similarly, using the fact that $0 \equiv(c a) b-c(a b)\left(\bmod J^{4}\right)$ we obtain the following equations:

$$
\begin{aligned}
& 0=\alpha_{6}+\gamma_{6}-\alpha_{4} \beta_{5}-\alpha_{7}+\alpha_{7} \beta_{5}, \\
& 0=\beta_{5} \gamma_{6}-\beta_{4} \beta_{5}-\beta_{7}+\beta_{5} \beta_{7}, \\
& 0=\beta_{6}+\gamma_{6}-\beta_{5} \gamma_{6}-1-\beta_{5} \gamma_{4}-\gamma_{7}+\beta_{5} \gamma_{7} .
\end{aligned}
$$

Now we add (4.10) and (4.11) and subtract (4.13) and (4.14) from the result to obtain $0=\left(\beta_{5}+1\right)\left(\beta_{4}-\beta_{6}+\gamma_{4}-\gamma_{6}\right)$. Hence $\beta_{5}=-1$. Then we subtract (4.12) from (4.9) and obtain $0=\gamma_{4}-\gamma_{6}-1$. Hence $\gamma_{6}=\gamma_{4}-1$. Next we subtract (4.14) from (4.11) and obtain $0=\beta_{4}-\beta_{6}$. Hence $\beta_{6}=\beta_{4}$. Then we use the fact that $b(b a) \equiv\left(b^{2}\right) a\left(\bmod J^{4}\right)$ to obtain that $\alpha_{7}=\alpha_{3}+\gamma_{3}, \beta_{7}=-\gamma_{3}$ and $\gamma_{7}=\beta_{3}+2 \gamma_{3}$. Now (4.10) implies that $\beta_{4}=\gamma_{4}-1-2 \gamma_{3}$. Using the fact that $0 \equiv\left(c^{2}\right) a-c(c a)\left(\bmod J^{4}\right)$ we obtain the following equations:

$$
\begin{aligned}
& 0=\beta_{3}-\gamma_{3}-3-4 \alpha_{3}+2 \alpha_{4}+2 \alpha_{6}, \\
& 0=4 \gamma_{4}-2 \beta_{3}-1-6 \gamma_{3} ;
\end{aligned}
$$

in particular, $\operatorname{char} F \neq 2$. Now (4.11) forces $0=4+2 \beta_{3}+6 \gamma_{3}-4 \gamma_{4}$, so $\beta_{3}=2 \gamma_{4}-3 \gamma_{3}-2$. Next we multiply (4.9) by 2 and subtract (4.15) to obtain $0=3$, so $\operatorname{char} F=3$. Thus

$$
\begin{gathered}
J^{2}=F\left\{[a, b],[a, c],[b, c],[a, a b],[a, a c],\left[b, a^{2}\right],\right. \\
\left.[b, a b],[b, a c],\left[c, a^{2}\right],[c, a b],[c, a c]\right\}+J^{4} \\
\subset F\left\{[a, b],[a, c],[b, c], a^{3}, a^{2} b-a^{2} c\right\}+J^{4}
\end{gathered}
$$

as is easily checked. But this contradicts the fact that $\operatorname{dim} J^{2} / J^{4}=6$.

Case 2. $\operatorname{dim} J^{3} / J^{4}=2$. We distinguish two more cases.

Case 2.1. $a^{2} b \in F a^{3}+J^{4}$. In this case $J^{3}=F\left\{a^{3}, a^{2} b, a^{2} c\right\}+J^{4}=F\left\{a^{3}, a^{2} c\right\}$ $+J^{4}$, and $a^{2} b \equiv \delta a^{3}\left(\bmod J^{4}\right)$ for some element $\delta \in F$. Since $a^{3} c \equiv a^{2} b a \equiv$ $\delta a^{4}\left(\bmod J^{5}\right)$ we see that $J^{4}=F a^{4}+F a^{3} c+J^{5}=F a^{4}+J^{5}$. Since $J^{4} \neq J^{5}$ this implies that $\operatorname{dim} J^{4} / J^{5}=1$. Now Lemma $G$ in [6] shows that $J^{3} \subset Z$; in particular, $a^{2} c \in Z$. But this leads to the contradiction $0 \equiv\left(a^{2} c\right) a-a\left(a^{2} c\right) \equiv$ $a^{2}(c a)-a^{3} c \equiv a^{4}\left(\bmod J^{5}\right)$.

Case 2.2. $a^{2} b \notin F a^{3}+J^{4}$. Since $a^{3} \notin J^{4}$ and $\operatorname{dim} J^{3} / J^{4}=2$ the elements $a^{3}+J^{4}$ and $a^{2} b+J^{4}$ form a basis of $J^{3} / J^{4}$ in this case. We write $a^{2} c \equiv$ $\delta a^{3}+\varepsilon a^{2} b\left(\bmod J^{4}\right)$ with elements $\delta, \varepsilon \in F$. Since $J^{4}=F a^{4}+F a^{3} b+J^{5}$ and $J^{4} \neq J^{5}$ we have $\operatorname{dim} J^{4} / J^{5} \in\{1,2\}$. Let us distinguish the corresponding cases.

Case 2.2.1. $\operatorname{dim} J^{4} / J^{5}=2$. In this case the elements $a^{4}+J^{5}$ and $a^{3} b+J^{5}$ form a basis of $J^{4} / J^{5}$. Since

$$
0 \equiv\left(a^{2} c\right) a-a^{2}(c a) \equiv\left(\delta \varepsilon+\beta_{5} \delta-1\right) a^{4}+(\varepsilon-1)\left(\varepsilon+\beta_{5}\right) a^{3} b \quad\left(\bmod J^{5}\right)
$$

this implies that $\delta \varepsilon+\beta_{5} \delta-1=0$ and $(\varepsilon-1)\left(\varepsilon+\beta_{5}\right)=0$. The first equation forces $\varepsilon \neq-\beta_{5}$, so $\varepsilon=1$ by the second equation. Then, using the fact that $0 \equiv(b c) a-b(c a)+c(b a)-(c b) a\left(\bmod J^{4}\right)$ we obtain the contradiction $0=$ $\left(1+\beta_{5}\right)\left(\beta_{4}-\beta_{6}+\gamma_{4}-\gamma_{6}\right)$. 
Case 2.2.2. $\operatorname{dim} J^{4} / J^{5}=1$. In this case $J^{3} \subset Z$ by Lemma $G$ in [6]; in particular, $a^{2} b, a^{2} c \in Z$. Hence $a^{3} b \equiv a^{2} b a \equiv a^{3} c\left(\bmod J^{5}\right)$ and $a^{3} b \equiv a^{3} c \equiv$ $a^{2} c a \equiv a^{4}+a^{3} b\left(\bmod J^{5}\right)$, so $a^{4} \in J^{5}$ and $J^{4}=F a^{3} b+J^{5}$. Furthermore, since $a^{3} b \equiv a^{3} c \equiv \varepsilon a^{3} b\left(\bmod J^{5}\right)$ we must have $\varepsilon=1$. Using the fact that $0 \equiv$ $(b c) a-b(c a)+c(b a)-(c b) a\left(\bmod J^{4}\right)$ we obtain $0=\left(1+\beta_{5}\right)\left(\beta_{4}-\beta_{6}+\gamma_{4}-\gamma_{6}\right)$, so $\beta_{5}=-1$. Similarly, using the fact that $0 \equiv\left(b^{2}\right) a-b(b a)\left(\bmod J^{4}\right)$ we obtain $\gamma_{7}=\beta_{3}+\gamma_{3}-\beta_{7}$. Then $0 \equiv\left(a^{2} c\right) b-b\left(a^{2} c\right) \equiv\left(a^{2} c\right) b-(b a) a c\left(\bmod J^{5}\right)$ implies that $\delta=1+\beta_{3}+\gamma_{3}-\beta_{4}-\gamma_{4}$. But now the fact that $0 \equiv a^{2}\left(c^{2}\right)-\left(a^{2} c\right) c$ $\left(\bmod J^{5}\right)$ leads to a contradiction.

\section{(4.17) Lemma. The case (4.6) does not occur.}

Proof. We assume the contrary and distinguish two cases.

Case 1. $\operatorname{dim} J^{3} / J^{4}=3$. In this case the elements $a^{3}+J^{4}, a^{2} b+J^{4}, a^{2} c+J^{4}$ form a basis of $J^{3} / J^{4}$. Since $\beta_{2} \neq 1$ and

$$
0 \equiv(b c) a-b(c a) \equiv\left(1-\beta_{2}^{2}\right) a^{3}+\beta_{4}\left(\beta_{2}-\beta_{2}^{2}\right) a^{2} b+\gamma_{4}\left(\beta_{2}-\beta_{2}^{2}\right) a^{2} c \quad\left(\bmod J^{4}\right)
$$

this implies that $\beta_{2}=-1$; in particular, $\operatorname{char} F \neq 2$. Hence $0=2 \beta_{4}=2 \gamma_{4}$, so $0=\beta_{4}=\gamma_{4}$. Then, using similarly the fact that $0 \equiv c(b a)-(c b) a\left(\bmod J^{4}\right)$ we obtain $0=\beta_{6}=\gamma_{6}$. But now the fact that $0 \equiv(b c) b-b(c b)\left(\bmod J^{4}\right)$ leads to a contradiction.

Case 2. $\operatorname{dim} J^{3} / J^{4}=2$. We distinguish two more cases.

Case 2.1. $a^{2} b \in F a^{3}+J^{4}$. In this case we have $J^{3}=F\left\{a^{3}, a^{2} b, a^{2} c\right\}+J^{4}=$ $F a^{3}+F a^{2} c+J^{4}$ and $J^{4}=F a^{4}+F a^{3} c+J^{5}$. Assume that $a^{4} \in J^{5}$. Then $J^{4}=F a^{3} c+J^{5}$; in particular, $\operatorname{dim} J^{4} / J^{5}=1$ since $J^{4} \neq J^{5}$. Hence Lemma $\mathrm{G}$ in [6] implies that $J^{3} \subset Z$; in particular, $a^{2} c \in Z$. But this leads to the contradiction $a^{3} c \equiv a^{2} c a \equiv \beta_{2} a^{3} c\left(\bmod J^{5}\right)$.

We write $a^{2} b \equiv \delta a^{3}\left(\bmod J^{4}\right)$ with some element $\delta \in F$. Then $\delta a^{4} \equiv$ $a^{2} b a \equiv \beta_{2} a^{3} b \equiv \beta_{2} \delta a^{4}\left(\bmod J^{5}\right)$. Since $a^{4} \notin J^{5}$ and $\beta_{2} \neq 1$ this implies that $\delta=0$. As in Case 1 , we now use the fact that $0 \equiv(b c) a-b(c a)\left(\bmod J^{4}\right)$ to obtain that $\beta_{2}=-1$, char $F \neq 2$ and $\gamma_{4}=0$. Similarly, using the fact that $0 \equiv(c b) a-c(b a) \equiv\left(b^{2}\right) a-b(b a)\left(\bmod J^{4}\right)$ we obtain $0=2 \gamma_{6}=2 \gamma_{3}$, so $0=\gamma_{6}=\gamma_{3}$. But this yields a contradiction using the fact that $0 \equiv(b c) c-b\left(c^{2}\right)$ $\left(\bmod J^{4}\right)$.

Case 2.2. $a^{2} b \notin F a^{3}+J^{4}$. Since $a^{3} \notin J^{4}$ and $\operatorname{dim} J^{3} / J^{4}=2$ the elements $a^{3}+J^{4}$ and $a^{2} b+J^{4}$ form a basis of $J^{3} / J^{4}$ in this case, and $J^{4}=F a^{4}+F a^{3} b+$ $J^{5}$. Assume that $a^{4} \in J^{5}$. Then $J^{4}=F a^{3} b+J^{5}$; in particular, $\operatorname{dim} J^{4} / J^{5}=1$ since $J^{4} \neq J^{5}$. Hence Lemma $G$ in [6] implies that $J^{3} \subset Z$; in particular, $a^{2} b \in Z$. But now we obtain the contradiction $a^{3} b \equiv a^{2} b a \equiv \beta_{2} a^{3} b\left(\bmod J^{5}\right)$.

Hence $a^{4} \notin J^{5}$, and we write $a^{2} c \equiv \delta a^{3}+\varepsilon a^{2} b\left(\bmod J^{4}\right)$ with elements $\delta, \varepsilon \in F$. Then $0 \equiv\left(a^{2} c\right) a-a^{2}(c a) \equiv\left(1-\beta_{2}\right) \delta a^{4}\left(\bmod J^{5}\right)$, so $\delta=0$ since $\beta_{2} \neq 1$ and $a^{4} \notin J^{5}$. As in Case 1 , we now use the fact that $0 \equiv(b c) a-b(c a)$ $\left(\bmod J^{4}\right)$ to obtain $\beta_{2}=-1$ and $\operatorname{char} F \neq 2$. Then we distinguish two more cases.

Case 2.2.1. $\operatorname{dim} J^{4} / J^{5}=2$. In this case the elements $a^{4}+J^{5}$ and $a^{3} b+J^{5}$ form a basis of $J^{4} / J^{5}$. Using the fact that $0 \equiv b\left(a^{2} c\right)-(b a) a c\left(\bmod J^{5}\right)$ 
we obtain $\alpha_{3} \varepsilon=1$. But this leads to a contradiction using the fact that $0 \equiv$ $\left(a^{2} c\right) b-a^{2}(c b)\left(\bmod J^{4}\right)$.

Case 2.2.2. $\operatorname{dim} J^{4} / J^{5}=1$. In this case we have $J^{4}=F a^{4}+J^{5}$ since $a^{4} \notin$ $J^{5}$. Moreover, Lemma G in [6] implies that $J^{3} \subset Z$; in particular, $a^{2} b \in Z$. Thus $a^{3} b \equiv a^{2} b a \equiv-a^{3} b\left(\bmod J^{5}\right)$, so $a^{3} b \in J^{5}$ since $\operatorname{char} F \neq 2$. This, however, leads to a contradiction using the fact that $0 \equiv\left(a^{2} c\right) b-b\left(a^{2} c\right) \equiv$ $a^{2}(c b)-(b a) a c\left(\bmod J^{5}\right)$.

\section{REFERENCES}

1. J. Brandt, $A$ lower bound for the number of irreducible characters in a block, J. Algebra 74 (1982), 509-514.

2. R. Brauer, Defect groups in the theory of representations of finite groups, Illinois J. Math. 13 (1969), 53-73.

3. M. Broue and L. Puig, A Frobenius theorem for blocks, Invent. Math. 56 (1981), 117-128.

4. B. Külshammer, On the structure of block ideals in group algebras of finite groups, Comm. Algebra 8 (1980), 1867-1872.

5. $\frac{1}{1-7}$, Bemerkungen über die Gruppenalgebra als symmetrische Algebra, J. Algebra 72 (1981), $1-7$.

6. __ Symmetric local algebras and small blocks of finite groups, J. Algebra 88 (1984), 190-195.

7. P. Landrock, On the number of irreducible characters in a 2-block, J. Algebra 68 (1981), 426-442.

Department of Mathematics, University of Dortmund, 4600 Dortmund 50, Federal REPUBLIC OF GERMANY

Current address: Department of Mathematics, University of Ausburg, 8900 Augsburg, Germany 